\title{
Two-chamber lattice model for thermodiffusion in polymer solutions
}

\author{
Jutta Luettmer-Strathmann* \\ Department of Physics, The University of Akron \\ Akron, OH 44325-4001
}

(Dated: November 14, 2018)

\begin{abstract}
When a temperature gradient is applied to a polymer solution, the polymer typically migrates to the colder regions of the fluid as a result of thermal diffusion (Soret effect). However, in recent thermodiffusion experiments on poly(ethylene-oxide) (PEO) in a mixed ethanol/water solvent it is observed that for some solvent compositions the polymer migrates to the cold side, while for other compositions it migrates to the warm side. In order to understand this behavior, we have developed a two-chamber lattice model approach to investigate thermodiffusion in dilute polymer solutions. For a short polymer chain in an incompressible, one-component solvent we obtain exact results for the partitioning of the polymer between a warm and a cold chamber. In order to describe mixtures of PEO, ethanol, and water, we have extended this simple model to account for compressibility and hydrogen bonding between PEO and water molecules. For this complex system, we obtain approximate results for the composition in the warmer and cooler chambers that allow us to calculate Soret coefficients for given temperature, pressure, and solvent composition. The sign of the Soret coefficient is found to change from negative (polymer enriched in warmer region) to positive (polymer enriched in cooler region) as the water content of the solution is increased, in agreement with experimental data. We also investigate the temperature dependence of the Soret effect and find that a change in temperature can induce a change in the sign of the Soret coefficient. We note a close relationship between the solvent quality and the partitioning of the polymer between the two chambers, which may explain why negative Soret coefficients for polymers are so rarely observed.
\end{abstract}

\section{INTRODUCTION}

A temperature gradient applied to a fluid mixture generally induces a net mass flow, which results in the formation of a concentration gradient. This effect is known as thermodiffusion or the Ludwig-Soret effect ${ }^{1,2,3,4}$ The Soret coefficient $S_{\mathrm{T}}$ relates the steady state concentration gradient to the imposed temperature gradient. By convention, the Soret coefficient of component $i$ is positive if component $i$ is enriched in the cooler region $\underline{\underline{5}}$ Thermal diffusion has long been used as an effective tool for separating mixtures of isotopes $\stackrel{4}{-}$ More recently, the effect has been used to characterize mixtures of complex fluids (see for example Refs. 5 6 6 7,8).

In liquid mixtures whose components differ widely in molecular mass, such as polymer solutions $\frac{6.7}{}$ and colloidal suspensions ${ }^{8}$, it is typically the heavier component that migrates to the cold region. There are, however, exceptions. In 1977, Giglio and Vendramini found a negative Soret coefficient for poly(vinyl alcohol) in water ${ }^{9}$ Very recently, de Gans et al ${ }^{10,11}$ reported results of thermal diffusion forced Raleigh scattering (TDFRS) measurements on solutions of poly(ethylene oxide) (PEO) in mixtures of ethanol and water. In pure water, $\mathrm{PEO}$ shows the expected migration to the cold region of the fluid $\left(S_{\mathrm{T}}>0\right)$. However, in solutions with low water content, PEO is found to migrate to the warmer region of the fluid $\left(S_{\mathrm{T}}<0\right)$. Although changes in sign of the Soret coefficient have been reported for a number of liquid mixtures of small-molecule fluids, including alcohol solutions, ${ }^{12.13 .14 .15}$ the $\mathrm{PEO} /$ ethanol/water system appears to be the first polymer solution for which such a sign change has been observed.
Thermodiffusion in a binary fluid mixture is described by the flux of one of the components in response to a temperature and concentration gradient $\underline{\underline{1}}$ The flux is given by

$$
J_{1}=-\rho D \nabla c_{1}-\rho c_{1}\left(1-c_{1}\right) D^{\prime} \nabla T,
$$

where $D$ is the mutual diffusion coefficient, $D^{\prime}$ the thermal diffusion coefficient of component $1, \rho$ the total mass density, $c_{1}$ the mass fraction of component 1 , and $T$ is the temperature. Here the pressure is assumed to be constant throughout the mixture and the flux $J_{1}$ describes the flow of component 1 with respect to the center of mass of the system $1 \underline{1}$ Eventually, the system reaches a stationary state in which the flux $J_{1}$ vanishes. Inserting $J_{1}=0$ into Eq. (11) yields

$$
-\frac{1}{c_{1}\left(1-c_{1}\right)} \frac{\nabla c_{1}}{\nabla T}=\frac{D^{\prime}}{D}
$$

The Soret coefficient of component 1 is the ratio of thermal and mutual diffusion coefficients

$$
S_{\mathrm{T}}=\frac{D^{\prime}}{D} .
$$

More generally, we define the Soret coefficient of component $i$ of a mixture as

$$
S_{\mathrm{T}}=-\frac{1}{c_{i}\left(1-c_{i}\right)} \frac{d c_{i}}{d T} .
$$

In order to describe thermodiffusion in ternary mixtures, such as PEO in a mixed solvent, concentration gradients and fluxes of two of the components are 
considered ${ }^{1.16}$

$$
\begin{aligned}
& J_{1}=-\rho D_{11} \nabla c_{1}-\rho D_{12} \nabla c_{2}-\rho c_{1}\left(1-c_{1}\right) D_{1}^{\prime} \nabla T, \\
& J_{2}=-\rho D_{21} \nabla c_{1}-\rho D_{22} \nabla c_{2}-\rho c_{2}\left(1-c_{2}\right) D_{2}^{\prime} \nabla T,
\end{aligned}
$$

where $J_{1}$ and $J_{2}$ are the flux of component 1 and 2, respectively, and the flux of the third component is given by $J_{3}=-J_{1}-J_{2}$. Due to Onsager's relations, only three of the four isothermal diffusion coefficients $D_{i j}$ are independent. Their relationship has a complicated form, however, since the fluxes are defined with respect to the barycentric reference frame ${ }^{1}$. The prefactors of the generalized thermal diffusion coefficients $D_{i}^{\prime}$ have been chosen in such a way that Eq. (11) for the binary mixture is recovered for $c_{2}=0$ or $c_{1}=0$. In the steady state, each of the fluxes vanishes and the relationship (2) between composition and temperature gradient generalizes to

$$
\begin{aligned}
& -\frac{1}{c_{1}\left(1-c_{1}\right)} \frac{\nabla c_{1}}{\nabla T}= \\
& \quad \frac{c_{1}\left(1-c_{1}\right) D_{22} D_{1}^{\prime}-c_{2}\left(1-c_{2}\right) D_{12} D_{2}^{\prime}}{c_{1}\left(1-c_{1}\right)\left(D_{11} D_{22}-D_{12} D_{21}\right)},
\end{aligned}
$$

and similarly for component 2. For ternary mixtures, we continue to define the Soret coefficient of component $i$ through Eq. (4).

Thermal diffusion in liquid mixtures is not well understood and even the sign of the Soret coefficient cannot generally be predicted (see e.g. Refs. 45 6). Due to the complexity of the task, attempts to extend the kinetic gas theory 17 of thermodiffusion to the liquid state have so far been unsuccessfu14.6. Molecular dynamics simulations (for a review see Ref. 18) have become an important tool in the investigation of thermodiffusion in small-molecule liquids. Long computation times make it difficult, however, to address thermodiffusion in polymeric systems.

Earlier theoretical work on thermodiffusion, see e.g. Refs. 1 2 3 4, 13 19 20 21 22 23, made use of the "heat of transport" concept. For dilute binary solutions, the Soret coefficient of the solute is related to the heats of transport of the two species as follows 23

$$
S_{\mathrm{T}}=\frac{Q_{1}^{*}-Q_{2}^{*}}{k_{\mathrm{B}} T^{2}}
$$

where $k_{\mathrm{B}}$ is Boltzmann's constant. The numerator represents the net energy flowing through the boundary of a small volume of the mixture when the composition inside the region changes while temperature and pressure remain constant ${ }^{2}$. Various microscopic expressions for the heat of transport have been obtained with the aid of lattice model calculations. Wirtz and Hiby $19,20,21$ considered two contributions to the energy required for a particle to move from one location to another, the energy to detach the particle from its neighbors and the energy to create a void for the particle to move into. Denbigh ${ }^{23}$ considered the energy associated with particle diffusion across a boundary to be a "certain fraction" of the change in potential energy, which is represented by nearest-neighbor interactions in randommixing approximation ${ }^{23}$ This type of approach is useful in describing thermal diffusion in regular solutions 4 but fails for more complex systems. Prigogine et al $l^{13}$ found that a model based on energies of nearest-neighbor interactions alone $\mathrm{e}^{22}$ led to contradictions when applied to alcohol mixtures, where the Soret coefficient changes sign ${ }^{12.13}$. They argued that a "free energy" for detaching a molecule needs to be considered in systems with associating solvents. By assuming that alcohol molecules form complexes whose disruption results in a loss of local energy and a gain in local entropy, they were able to explain qualitatively the sign change of the Soret coefficient for these systems $\underline{\underline{13}}$

In this work, we investigate the Soret effect in dilute polymer solutions with the aid of two-chamber lattice models. Following traditional experimental methods $\stackrel{1,2,3.4}{2}$ we consider a system divided into two chambers of equal size that are maintained at slightly different temperatures. Particles are free to move between the chambers, which do not otherwise interact. If the pressure differences between the chambers are small enough to be neglected, the Soret coefficient can be determined from the difference in composition of the solutions in the two chambers $\frac{1.2 .3 .4}{1}$ We start by investigating a polymer chain in an incompressible, one-component solvent in Section III This allows us to introduce the general strategy and obtain exact results for a simple model system. Section III focuses on $\mathrm{PEO} /$ ethanol/water mixtures. In Section IIA we present a lattice model for the static properties that includes the effects of compressibility and specific interactions between PEO and water. The corresponding two-chamber system is introduced in Section IIIB Results of our calculations are presented in Section IV and compared with experimental data, where available. In Section $\nabla$ we discuss the work presented here and provide an outlook to future work. The appendices describe the determination of system-dependent parameters for $\mathrm{PEO} /$ ethanol/water mixtures and the relation between physical properties and model variables.

\section{LATTICE MODEL FOR INCOMPRESSIBLE, DILUTE POLYMER SOLUTIONS}

\section{A. Single chamber}

We consider a simple cubic lattice of $N$ sites. Since we are interested in dilute solutions, we assume that only one polymer chain is present. A chain of $N_{\mathrm{c}}$ beads has $N_{\mathrm{c}}-1$ bonds and occupies without overlap $N_{\mathrm{c}}$ contiguous lattice sites. For an incompressible system, all of the remaining lattice sites are occupied individually and represent solvent molecules. The filling fraction of the polymer and the solvent are defined as $\phi_{\mathrm{p}}=N_{\mathrm{c}} / N$ and $\phi_{\mathrm{s}}=N_{\mathrm{s}} / N$, respectively, with $\phi_{\mathrm{s}}=1-\phi_{\mathrm{p}}$. Interactions between non-bonded chain segments on nearest-neighbor lattice sites are described by an interaction energy $\epsilon_{\mathrm{pp}}$. 


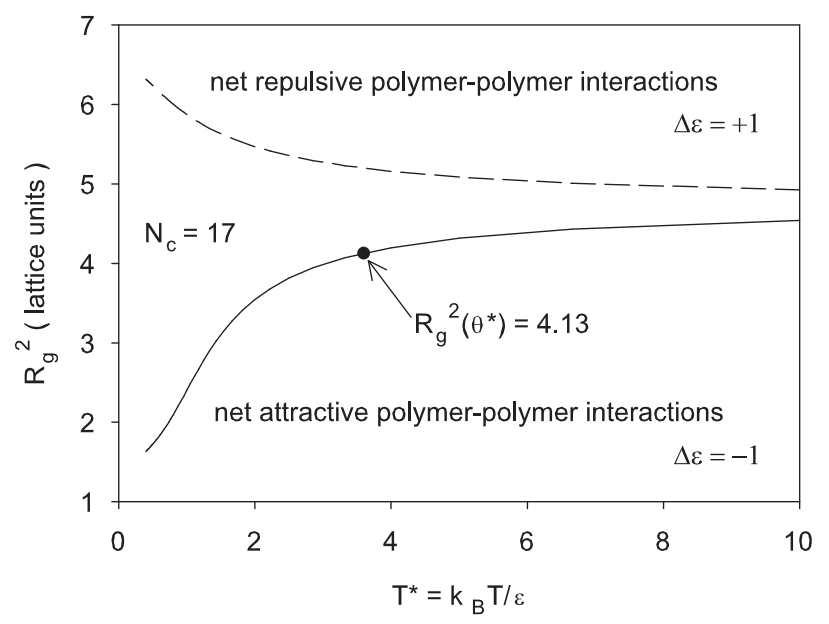

FIG. 1: Radius of gyration squared of chains of length $N_{\mathrm{c}}=17$ as a function of reduced temperature for net attractive $(\Delta \epsilon<0)$ and net repulsive $(\Delta \epsilon>0)$ polymer-polymer interactions.

Polymer-solvent and solvent-solvent interactions are also restricted to nearest-neighbor lattice sites and described by interaction energies $\epsilon_{\mathrm{ps}}$ and $\epsilon_{\mathrm{ss}}$, respectively.

The number of chain conformations is given by the number of self-avoiding random walks of $N_{\mathrm{c}}-1$ steps and can be enumerated exactly for short chains (see e.g. Refs. 24, 25). The probability for a given chain conformation to be realized depends on the interactions in the system. For a chain conformation with $m$ pair contacts between polymer segments, the internal energy of the polymer-solvent system is given by

$$
\begin{aligned}
E(m)= & \left(m-\left(\frac{z}{2}-1\right) N_{\mathrm{c}}-1\right) \Delta \epsilon \\
& +\frac{z}{2}\left(N-N_{\mathrm{c}}\right) \epsilon_{\mathrm{ss}}+\left(\left(\frac{z}{2}-1\right) N_{\mathrm{c}}+1\right) \epsilon_{\mathrm{pp}}
\end{aligned}
$$

where $z=6$ is the coordination number of the simple cubic lattice and $\Delta \epsilon=\epsilon_{\mathrm{pp}}+\epsilon_{\mathrm{ss}}-2 \epsilon_{\mathrm{ps}}$ is the net interaction energy between polymer segments. The canonical partition function of the system is given by

$$
Z_{\mathrm{pol}}(T)=N \sum_{m} c(m) \exp (-\beta E(m)),
$$

where $c(m)$ is the number of chain conformations with $m$ polymer-polymer contacts and $\beta=1 / k_{\mathrm{B}} T$, where $T$ is the temperature and $k_{\mathrm{B}}$ is Boltzmann's constant. The internal energy for a given temperature is calculated in the usual way

$$
\begin{aligned}
U_{\mathrm{pol}} & =N \sum_{m} c(m) E(m) \exp (-\beta E(m)) / Z_{\mathrm{pol}} \\
& =k_{\mathrm{B}} T^{2} \frac{\partial \ln \left(Z_{\mathrm{pol}}\right)}{\partial T} .
\end{aligned}
$$

In a preliminary step, we generated all single chain conformations of chains up to length $N_{\mathrm{c}}=17$ and determined the number $m$ of polymer-polymer contacts for
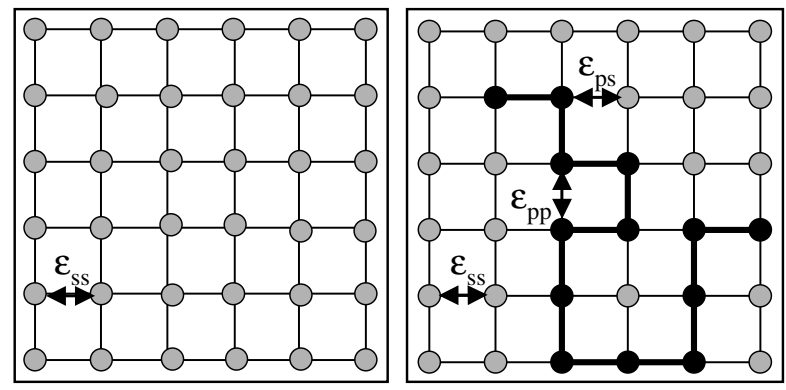

FIG. 2: Two-dimensional illustration of the two-chamber lattice model for the incompressible case. The right chamber contains the polymer chain, where the black circles and heavy lines indicate the beads and bonds of the polymer chain while the grey circles represent solvent sites.

each conformation as well as the radius of gyration. The radius of gyration for a given temperature is calculated from 24.25

$$
\left\langle R_{g}^{2}(T)\right\rangle=N \sum_{m} c(m) \bar{R}_{g}^{2}(m) \exp (-\beta E(m)) / Z_{\mathrm{pol}},
$$

where $\bar{R}_{g}^{2}(m)$ is the average radius of gyration squared for conformations with $m$ contacts. For the incompressible system, we express all interaction energies in terms of a positive energy unit $\epsilon$, which also sets the temperature scale; the reduced temperature $T^{*}$ is given by $T^{*}=k_{\mathrm{B}} T / \epsilon$. In Fig. 1 we present chain dimensions for chains of length $N_{\mathrm{c}}=17$ as a function of reduced temperature for the case of net attraction $(\Delta \epsilon<0)$ and net repulsion $(\Delta \epsilon>0)$ between the polymer segments. As expected, the chain expands with increasing temperature for the case $\Delta \epsilon<0$, while the chain decreases in size with increasing temperature for the case $\Delta \epsilon>0$.

For polymers in dilute solutions, the chain dimensions are an indicator for solvent quality ${ }^{26}$; the better the solvent the larger the chain dimensions. For very long chains with net attractive interactions between the polymer segments, a collapse transition is observed at the $\theta$ temperature of the polymer solution 26 . Monte Carlo simulations 27 yield an estimate of $\theta^{*}=3.60 \pm 0.05$ for the reduced $\theta$ temperature of an infinitely long isolated chain. From our exact enumeration results we determined the dimensions of an isolated chain of length $N_{\mathrm{c}}=17$ at the $\theta$ temperature of the infinite chain and found $R_{g}^{2}\left(\theta^{*}\right)=4.13$ in units of lattice constant squared.

\section{B. Two-chamber system}

In order to investigate thermodiffusion, consider a single-chain system that is divided into two chambers, A and B, with slightly different temperatures, $T^{\mathrm{A}}>T^{\mathrm{B}}$, see Fig. 2 Under the assumption that the chambers are non-interacting, the partition function of the whole system is the product of the partition functions of the indi- 
vidual chambers, $Z^{\mathrm{A}} Z^{\mathrm{B}}$. The chambers are represented by lattices with $N_{a}, a \in\{A, B\}$ sites. The canonical partition function of the chamber that contains the polymer is given by Eq. (10), the canonical partition function for the chamber without polymer consists of a single term

$$
Z_{\mathrm{nop}}\left(T_{a}\right)=\exp \left(-\beta_{a} E_{\mathrm{nop}}\right)
$$

with $a \in\{A, B\}$ and $E_{\text {nop }}=\frac{z}{2} N_{a} \epsilon_{\mathrm{ss}}$. The internal energy in this case is independent of temperature, $U_{\text {nop }}=$ $E_{\text {nop. }}$. If the polymer chain is allowed to be in either chamber, the sum of states for the system is given by

$$
Q=Z_{\mathrm{pol}}\left(T_{\mathrm{A}}\right) Z_{\mathrm{nop}}\left(T_{\mathrm{B}}\right)+Z_{\mathrm{pol}}\left(T_{\mathrm{B}}\right) Z_{\mathrm{nop}}\left(T_{\mathrm{A}}\right) .
$$

Accordingly, the probability $q_{\mathrm{A}}$ to find the polymer in the (warmer) chamber A can be calculated from

$$
q_{\mathrm{A}}=Z_{\mathrm{pol}}\left(T_{\mathrm{A}}\right) Z_{\mathrm{nop}}\left(T_{\mathrm{B}}\right) / Q .
$$

$q_{\mathrm{B}}=1-q_{\mathrm{A}}$ is the probability to find the chain in the cooler chamber B.

For small temperature differences $\delta T=T_{\mathrm{A}}-T_{\mathrm{B}}$ we set $T_{\mathrm{B}}=T, T_{\mathrm{A}}=T+\delta T$ and rewrite Eq. (16) as

$$
\frac{1}{q_{\mathrm{A}}}=1+\frac{Z_{\mathrm{pol}}(T) Z_{\mathrm{nop}}(T+\delta T)}{Z_{\mathrm{pol}}(T+\delta T) Z_{\mathrm{nop}}(T)} .
$$

Expanding the partition functions according to

$$
\ln (Z(T+\delta T))=\ln (Z(T))+\beta U(T) \delta T / T,
$$

where $\beta=1 / k_{\mathrm{B}} T$, we find for the probability

$$
\frac{1}{q_{\mathrm{A}}}=1+\exp \left(\beta\left(U_{\mathrm{nop}}-U_{\mathrm{pol}}\right) \frac{\delta T}{T}\right) .
$$

We employ temperature differences $\delta T / T=10^{-4}$ in our calculations and find the results from Eqs. (16) and (19) to be indistinguishable. In order to compare with the theories based on the heat of transport, 1.2.3.4.13.19.20.21.22.23 we expand the expression for the probability $q_{\mathrm{A}}-1 / 2$ from Eq. (19) in powers of $\delta T / T$ and obtain to first order

$$
q_{\mathrm{A}}-\frac{1}{2} \simeq-\frac{1}{4} \beta\left(U_{\mathrm{nop}}-U_{\mathrm{pol}}\right) \frac{\delta T}{T} .
$$

For this simple model, the probability to find the polymer in the warmer chamber $\mathrm{A}$ is larger than $1 / 2$ when the internal energy of the chamber without polymer is smaller than the internal energy of the chamber with polymer at temperature $T$. Hence, the difference in internal energy between two chambers, one with and one without polymer, at the same temperature determines the probability to find the polymer in the warmer of two chambers.

The Soret coefficient ( Eq. (4) ) can be calculated from the filling fraction as well as from the mass fraction, since $\partial c_{1} / \partial \phi_{1}=c_{1} c_{2} / \phi_{1} \phi_{2}$. Setting $\phi_{1}=\phi_{\mathrm{p}}$, we obtain in the dilute limit

$$
S_{\mathrm{T}} \simeq-\frac{1}{\phi_{\mathrm{p}}} \frac{d \phi_{\mathrm{p}}}{d T} \simeq-\frac{1}{\phi_{\mathrm{p}}} \frac{\left(\phi_{\mathrm{pA}}-\phi_{\mathrm{pB}}\right)}{\left(T_{\mathrm{A}}-T_{\mathrm{B}}\right)},
$$

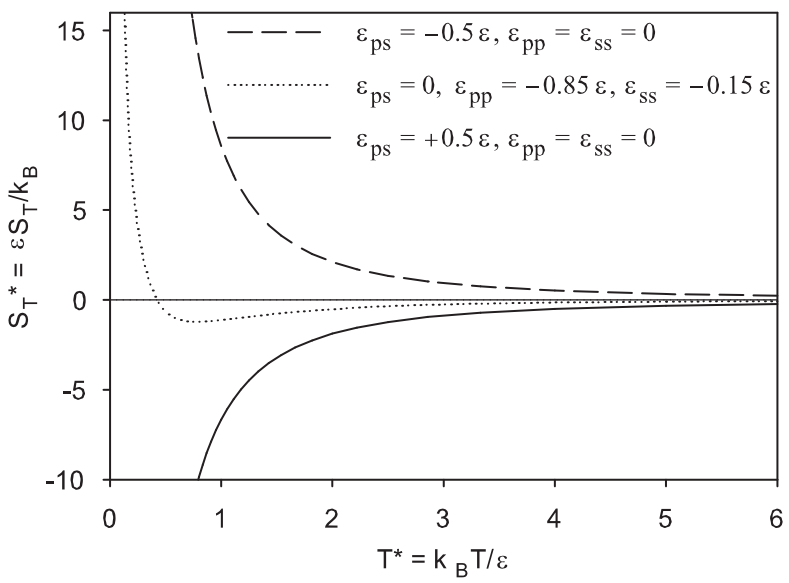

FIG. 3: Soret coefficients of an incompressible dilute polymer solution as a function of temperature. The curves represent exact enumeration results for chains of length $N_{\mathrm{c}}=17$, where the Soret coefficient has been calculated from Eq. (23). The interaction parameters are as indicated in the figure and correspond to net repulsive interactions $(\Delta \epsilon=+1)$ for the dashed curve and net attractive interactions $(\Delta \epsilon=-1)$ for the dotted and solid curves, respectively.

where the filling fractions in the chambers are calculated from the probability $q_{\mathrm{A}}$

$$
\phi_{\mathrm{pA}}=q_{A} N_{\mathrm{c}} / N_{\mathrm{A}}, \quad \phi_{\mathrm{pB}}=\left(1-q_{A}\right) N_{\mathrm{c}} / N_{\mathrm{B}} .
$$

Typically we assume that the chambers are equal in size and set $N_{\mathrm{A}}=N_{\mathrm{B}}=N / 2$, which yields for the Soret coefficient

$$
S_{\mathrm{T}}=-4 \frac{q_{\mathrm{A}}-\frac{1}{2}}{\delta T} .
$$

With the aid of Eq. (20) for the probability, the Soret coefficient becomes

$$
S_{\mathrm{T}}=\frac{U_{\mathrm{nop}}-U_{\mathrm{pol}}}{k_{\mathrm{B}} T^{2}} .
$$

In agreement with the definition of the Soret coefficient, this expression for $S_{\mathrm{T}}$ is independent of the temperature difference $\delta T$. It is also independent of the polymer concentration, in agreement with experimental results for dilute polymer solutions (cf. Ref. 28). Comparing Eq. (24) with the heat of transport expression for dilute solutions in Eq. (8), we see that the internal energy difference $U_{\text {nop }}-U_{\text {pol }}$ takes the place of the heat of transport difference $Q_{1}^{*}-Q_{2}^{*}$.

Results for the Soret coefficient calculated from Eqs. (19) and (23) are presented in Fig. 3. We note first that both positive and negative Soret coefficients are realized and that $S_{\mathrm{T}}$ may change sign as a function of temperature. For the simple model discussed in this section, the internal energy $U_{\text {nop }}$ of a solventfilled lattice is independent of temperature. If $U_{\mathrm{pol}}$ was also independent of temperature, then $S_{\mathrm{T}}$ would vary as 
$1 / T^{2}$, according to Eq. (24). The deviation of the actual calculated Soret coefficients from this $1 / T^{2}$ dependence, most notably the sign change of $S_{\mathrm{T}}$, is due to the temperature variation of the internal energy $U_{\mathrm{pol}}$ of the polymer system. A random mixing approximation for polymer-polymer and polymer-solvent contacts yields a temperature-independent internal energy $U_{\text {pol }}$. Hence, in order to calculate Soret coefficients of polymer solutions, one has to go beyond the random mixing approximation, as we do in this work.

A comparison of the results for the chain dimensions in Fig. 1 with the results for the Soret coefficients represented by the dashed and solid curves in Fig. [3 shows that the Soret coefficient decreases as the solvent quality increases. We find this trend confirmed when we consider the more complex system below. However, the Soret coefficients represented by the dotted curve show that this need not be the case. The chain dimensions for this case are the same as for the case represented by the solid line, since they correspond to the same net interaction energy $\Delta \epsilon=-1$. It was recognized early ${ }^{22.23}$ that, in contrast to thermodynamic and configurational properties, thermodiffusion is not determined by the net interaction energy $\Delta \epsilon$ but by all three interaction parameters. In fact, the simple heat of transfer models ${ }^{21,22.23}$ are most successful for regular solutions, where $\Delta \epsilon=0$.

\section{LATTICE MODEL FOR PEO IN ETHANOL/WATER MIXTURES}

Solutions of high molecular weight poly(ethylene oxide) (PEO) in ethanol and water have interesting properties. Hydrogen bonding between PEO and water molecules plays an important role in aqueous solutions of PEO (see e.g. Refs. 29 30). Water is a good solvent for PEO at standard temperature and pressure. However, the solvent quality decreases with temperature and a miscibility gap opens above a lower critical solution temperature 30 Ethanol, on the other hand, is a poor solvent for PEO at room temperature but the solubility increases with temperature $\stackrel{10}{\underline{10}}$. In mixtures of ethanol and water at standard temperature and pressure, the water content determines the solubility of PEO. For the molecular weight considered in this work, the transition between poor and good solvent condition appears between a water content of $5 \%$ and $10 \%$ by weight 10,11 . Light scattering experiments $\frac{10}{10}$ show that the PEO chains expand with increasing water content, indicating that the addition of water improves the solvent quality.

\section{A. Static properties}

In order to describe dilute solutions of poly(ethylene oxide) (PEO) in mixtures of ethanol and water, we extend the lattice model introduced above. At a given temperature, pressure, and composition, the single chamber system is represented by a simple cubic lattice with $N$ sites, of which $N_{\mathrm{c}}, N_{\mathrm{s}}$, and $N_{\mathrm{w}}$ are occupied by the polymer (PEO), the first solvent (ethanol), and the second solvent (water), respectively. In order to account for compressibility, we allow sites to be unoccupied so that $N=N_{\mathrm{c}}+N_{\mathrm{s}}+N_{\mathrm{w}}+N_{\mathrm{v}}$, where $N_{\mathrm{v}}$ is the number of voids. The total volume of the lattice is $V=v_{0} N$, where $v_{0}$ is the volume of one elementary cube.

Interactions between occupied nearest neighbor sites are described by interaction energies $\epsilon_{i j}$, where the subscripts indicate the occupants of the sites (p for polymer, $\mathrm{s}$ and $\mathrm{w}$ for the solvents; voids are assumed to have zero interaction energies). In aqueous solutions, hydrogen bonding between PEO and water plays an important role (cf. Ref. 29). In order to account for these specific interactions, we introduce an orientational degree of freedom in the description of water. Each elementary cube occupied by water is assumed to have one special face. If this face is exposed to a polymer segment, the interaction energy is $\epsilon_{\mathrm{pw} ; \mathrm{s}}$ (strongly attractive) otherwise $\epsilon_{\mathrm{pw} ; \mathrm{n}}$ (non-specific).

Because of the complexity of the system, we can no longer evaluate the canonical partition function exactly. In the following, we employ the exact enumeration results for the chains of length $N_{\mathrm{c}}=17$ and consider explicitly contacts that involve the polymer but we employ a random mixing approximation for all other contacts. A chain conformation with $m$ pair contacts has $n_{\mathrm{n}}=4 N_{\mathrm{c}}+2-2 m$ nearest neighbor $(\mathrm{nn})$ sites, which are occupied by $n_{i}, i \in\{\mathrm{s}, \mathrm{w}, \mathrm{v}\}$ solvent particles and voids. With the aid of the random mixing approximation for all but the polymer contacts, the canonical partition function of the system can be written as

$$
\begin{aligned}
& Z_{\mathrm{pol}}\left(N, T, N_{\mathrm{w}}, N_{\mathrm{s}}\right)= \\
& \quad N \sum_{m} c(m) \sum_{\left[n_{\mathrm{w}}\right]} 6^{N_{\mathrm{w}}-n_{\mathrm{w}}}\left(\begin{array}{c}
n_{\mathrm{n}} \\
n_{\mathrm{w}}
\end{array}\right)\left(\begin{array}{c}
N-n_{\mathrm{n}}-N_{\mathrm{c}} \\
N_{\mathrm{w}}-n_{\mathrm{w}}
\end{array}\right) \\
& \quad \times \sum_{\left[n_{\mathrm{s}}\right]}\left(\begin{array}{c}
n_{\mathrm{n}}-n_{\mathrm{w}} \\
n_{\mathrm{s}}
\end{array}\right)\left(\begin{array}{c}
N-n_{\mathrm{n}}-N_{\mathrm{c}}-\left(N_{\mathrm{w}}-n_{\mathrm{w}}\right) \\
N_{\mathrm{s}}-n_{\mathrm{s}}
\end{array}\right) \\
& \quad \times e^{-\beta\left(m \epsilon_{\mathrm{pp}}+n_{\mathrm{s}} \epsilon_{\mathrm{ps}}\right)}\left(5 e^{-\beta \epsilon_{\mathrm{pw} ; \mathrm{n}}}+e^{-\beta \epsilon_{\mathrm{pw} ; \mathrm{s}}}\right)^{n_{\mathrm{w}}} e^{-\beta E_{\mathrm{r}}}
\end{aligned}
$$

where, as before, $c(m)$ is the number of chain conformations with $m$ polymer-polymer contacts. The square brackets around the summation indices indicate that the summation is performed consistent with the available nearest neighbor sites and the total filling of the lattice. The energy $E_{\mathrm{r}}$ denotes the contribution to the total energy due to solvent-solvent interactions evaluated in random mixing approximation ${ }^{31}$, cf. Eq. (29) below.

The pressure of the system is calculated from

$$
P=\frac{k_{\mathrm{B}} T}{v_{0}}\left(\frac{\partial \ln Z_{\mathrm{pol}}}{\partial N}\right)_{N_{\mathrm{s}}, N_{\mathrm{w}}, N_{\mathrm{c}}} .
$$

By performing partial summations over the terms in Eq. (25) the probabilities for specific sets of states can 

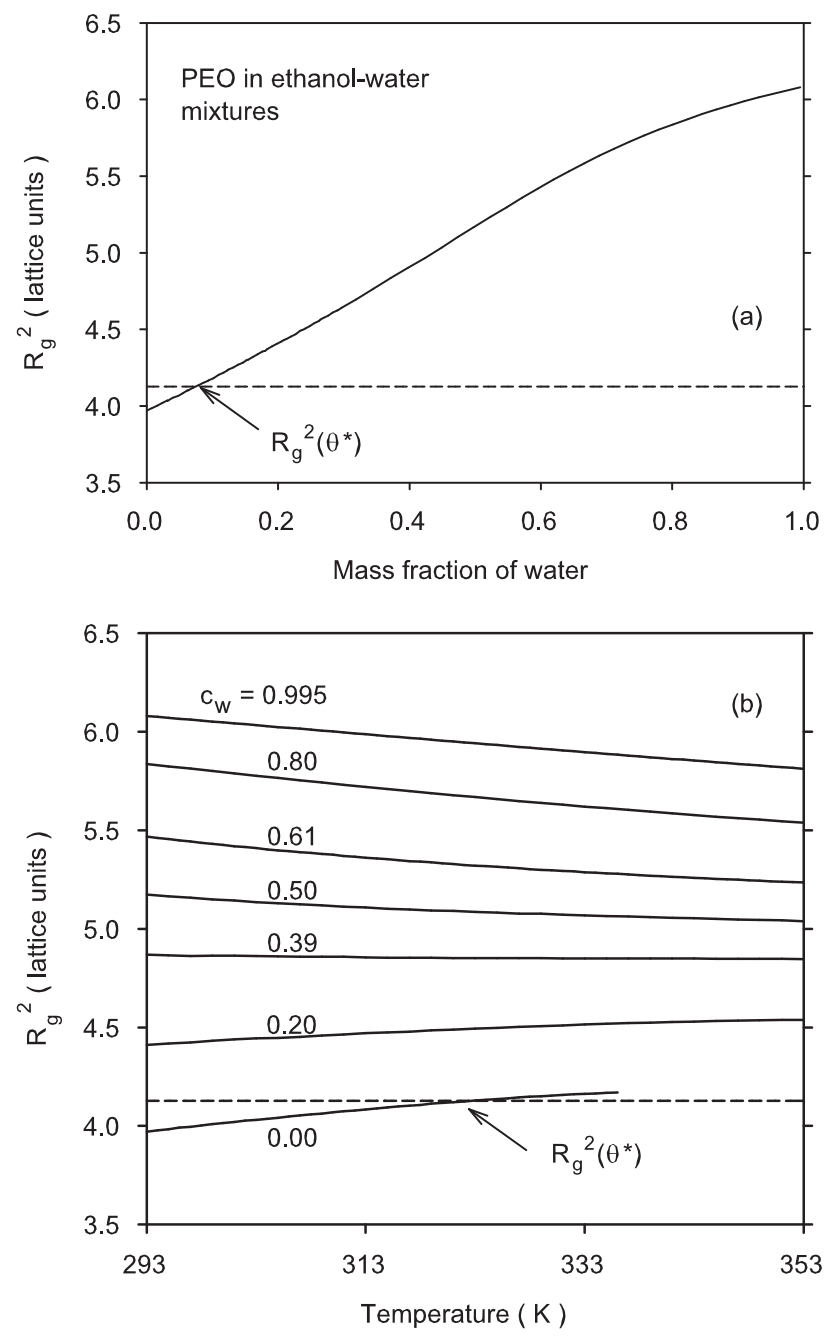

FIG. 4: Radius of gyration squared, $R_{\mathrm{g}}^{2}$, as calculated from Eq. (28). Panel (a) shows chain dimensions as a function of solvent composition at temperature $T=293 \mathrm{~K}$, pressure $P \approx 0.1 \mathrm{MPa}$, and a PEO concentration of $5 \mathrm{~g} / \mathrm{L}$. Panel (b) shows the temperature variation of the chain dimensions when the solutions are heated at constant pressure. The dashed line indicates the chain dimensions, $R_{\mathrm{g}}^{2}\left(\theta^{*}\right)$, of the isolated chain at the $\theta$ temperature of the infinite chain, as discussed in Section IIA

be determined. If we write the partition function as

$$
Z_{\mathrm{pol}} \equiv \sum_{m,\left[n_{\mathrm{w}}\right],\left[n_{\mathrm{s}}\right]} Z_{m, n_{\mathrm{w}}, n_{\mathrm{s}}}
$$

the average radius of gyration $\left\langle R_{\mathrm{g}}^{2}\right\rangle$ is given by

$$
\left\langle R_{\mathrm{g}}^{2}\right\rangle=Z_{\mathrm{pol}}^{-1} \sum_{m,\left[n_{\mathrm{w}}\right],\left[n_{\mathrm{s}}\right]} \bar{R}_{\mathrm{g}}^{2}(m) Z_{m, n_{\mathrm{w}}, n_{\mathrm{s}}} .
$$

In Appendix $\$$ we discuss how the system-dependent parameters of the lattice model were determined. In Appendix $\mathrm{B}$ we describe how lattice size and occupation numbers for given temperature, pressure and composi- tion of the mixture are calculated. Since chain dimensions are an indicator for solvent quality, we employed calculated $R_{\mathrm{g}}^{2}$ values to obtain estimates for the mixed interaction parameters. In Fig. 1 we present graphs for the chain dimensions calculated with the aid of the systemdependent parameters presented in Table [

\section{B. Two-chamber system}

As in section 【B we consider a system of two chambers with slightly different temperatures and determine the probability to find the polymer in the warmer of the two chambers. Assuming again that the chambers are non-interacting, the canonical partition function of the system for a given occupation of the chambers is the product of the individual partition functions.

To find the required canonical partition function of a chamber without polymer, consider a lattice of $N$ sites occupied by the two types of solvent and voids, $N=$ $N_{\mathrm{s}}+N_{\mathrm{w}}+N_{\mathrm{v}}$. Denoting the filling fractions of ethanol and water by $\phi_{\mathrm{s}}=N_{\mathrm{s}} / N$ and $\phi_{\mathrm{w}}=N_{\mathrm{w}} / N$ and assuming random mixing, the internal energy is given by ${ }^{31}$

$$
E_{\mathrm{nop}}=\frac{z}{2} N\left(\epsilon_{\mathrm{ss}} \phi_{\mathrm{s}}^{2}+\epsilon_{\mathrm{ww}} \phi_{\mathrm{w}}^{2}+2 \epsilon_{\mathrm{ws}} \phi_{\mathrm{s}} \phi_{\mathrm{w}}\right) .
$$

Accordingly, the canonical partition function of the lattice without polymer takes the form

$$
\begin{aligned}
Z_{\text {nop }}\left(N, T, N_{\mathrm{s}}, N_{\mathrm{w}}\right) \\
\quad=6^{N_{\mathrm{w}}}\left(\begin{array}{c}
N \\
N_{\mathrm{w}}
\end{array}\right)\left(\begin{array}{c}
N-N_{\mathrm{w}} \\
N_{\mathrm{s}}
\end{array}\right) e^{-\beta E_{\text {nop }}} .
\end{aligned}
$$

To ease notation for the two-chamber sum of states, we define the canonical partition for a single chamber as

$$
\begin{aligned}
Z(N, T, & \left.N_{\mathrm{w}}, N_{\mathrm{s}}, N_{\mathrm{p}}\right) \\
\quad & \left\{\begin{array}{lll}
Z_{\mathrm{pol}}\left(N, T, N_{\mathrm{s}}, N_{\mathrm{w}}\right) & \text { for } & N_{\mathrm{p}}=1 \\
Z_{\mathrm{nop}}\left(N, T, N_{\mathrm{s}}, N_{\mathrm{w}}\right) & \text { for } & N_{\mathrm{p}}=0
\end{array}\right.
\end{aligned}
$$

where $N_{\mathrm{p}} \in\{0,1\}$ is the number of polymer chains in the chamber.

For the mixed solvent system considered here, the concentration of the two solvents will generally be different in the warm and cold regions of a fluid. Since we have no a priori information about concentrations (or chemical potentials) of the solvents, we consider all distributions of particles consistent with fixed total particle numbers. The sum of states is then given by

$$
\begin{aligned}
Q & =\sum_{N_{\mathrm{p}, \mathrm{A}}=0}^{1} \sum_{\left[N_{\mathrm{w}, \mathrm{A}}\right]} \sum_{\left[N_{\mathrm{s}, \mathrm{A}}\right]} Z\left(N_{\mathrm{A}}, T_{\mathrm{A}}, N_{\mathrm{w}, \mathrm{A}}, N_{\mathrm{s}, \mathrm{A}}, N_{\mathrm{p}, \mathrm{A}}\right) \\
& \times Z\left(N-N_{\mathrm{A}}, T_{\mathrm{B}}, N_{\mathrm{w}}-N_{\mathrm{w}, \mathrm{A}}, N_{\mathrm{s}}-N_{\mathrm{s}, \mathrm{A}}, 1-N_{\mathrm{p}, \mathrm{A}}\right),
\end{aligned}
$$

where, as before, square brackets indicate summations consistent with the total numbers of particles and lattice sites. Also as before, chamber $\mathrm{A}$ is considered the 
warmer chamber so that $\delta T=T_{\mathrm{A}}-T_{\mathrm{B}}>0$, and equalsized chambers are used, $N_{\mathrm{A}}=N_{\mathrm{B}}=N / 2$. As we are performing the calculation of the terms in the sum of states, we monitor for each chamber the composition and the pressure of the mixtures. This allows us to calculate the average quantities for each chamber by performing weighted sums. For example, the average mass fraction of component $i$ in chamber $\mathrm{A}$, is calculated from

$$
\begin{aligned}
c_{i, \mathrm{~A}} & =\frac{1}{Q} \sum_{N_{\mathrm{p}, \mathrm{A}}=0}^{1} \sum_{\left[N_{\mathrm{w}, \mathrm{A}}\right]} \sum_{\left[N_{\mathrm{s}, \mathrm{A}}\right]} c_{i}\left(N_{\mathrm{w}, \mathrm{A}}, N_{\mathrm{s}, \mathrm{A}}, N_{\mathrm{p}, \mathrm{A}}\right) \\
& \times Z\left(N_{\mathrm{A}}, T_{\mathrm{A}}, N_{\mathrm{w}, \mathrm{A}}, N_{\mathrm{s}, \mathrm{A}}, N_{\mathrm{p}, \mathrm{A}}\right) \\
& \times Z\left(N-N_{\mathrm{A}}, T_{\mathrm{B}}, N_{\mathrm{w}}-N_{\mathrm{w}, \mathrm{A}}, N_{\mathrm{s}}-N_{\mathrm{s}, \mathrm{A}}, 1-N_{\mathrm{p}, \mathrm{A}}\right)
\end{aligned}
$$

where $c_{i}$ is the mass fraction of component $i$ calculated according to Eq. (B4).

The probability to find the polymer in chamber $\mathrm{A}$ in this model is given by

$$
\begin{aligned}
q_{\mathrm{A}} & =\frac{1}{Q} \sum_{\left[N_{\mathrm{w}, \mathrm{A}}\right]} \sum_{\left[N_{\mathrm{s}, \mathrm{A}}\right]} Z_{\mathrm{pol}}\left(N_{\mathrm{A}}, T_{\mathrm{A}}, N_{\mathrm{w}, \mathrm{A}}, N_{\mathrm{s}, \mathrm{A}}\right) \\
& \times Z_{\mathrm{nop}}\left(N-N_{\mathrm{A}}, T_{\mathrm{B}}, N_{\mathrm{w}}-N_{\mathrm{w}, \mathrm{A}}, N_{\mathrm{s}}-N_{\mathrm{s}, \mathrm{A}}\right) .
\end{aligned}
$$

As in the case of the incompressible system, this probability is related to an internal energy difference of two chambers at the same temperature:

$$
q_{\mathrm{A}}-\frac{1}{2} \simeq-\frac{1}{4} \frac{\left\langle U_{\mathrm{nop}}\right\rangle-\left\langle U_{\mathrm{pol}}\right\rangle}{k_{\mathrm{B}} T} \frac{\delta T}{T} .
$$

The angular brackets indicate an average over all configurations of particles in two chambers at the same temperature $T$, where the polymer is confined to one of the chambers. $U_{\text {pol }}$ and $U_{\text {nop }}$ are the average internal energies of the chamber with and without polymer at fixed composition. For the small temperature differences $\delta T=10^{-4} \mathrm{~K}$, corresponding to $\delta T / T \simeq 3 \times 10^{-7}$, employed in our calculations, values for the probability $q_{\mathrm{A}}$ calculated from Eqs (34) and (35) agree to more than five digits. As in the incompressible case, the excess probability $q_{\mathrm{A}}-1 / 2$ is proportional to the temperature difference and independent of the polymer concentration for dilute solutions. Relations similar to Eq. (35) can be derived for the probability of finding a given number of solvent particles in the warmer chamber A. They show that the difference between the average numbers of particles in chambers $\mathrm{A}$ and $\mathrm{B}$ is proportional to $\delta T$.

\section{RESULTS}

We have applied our lattice model to solutions of PEO in ethanol/water mixtures under a variety of conditions. As noted above, calculated values for composition differences in the two chambers are expected to be proportional to the temperature difference $\delta T=T_{\mathrm{A}}-T_{\mathrm{B}}$ for sufficiently small values of $\delta T$. We find this to be the case

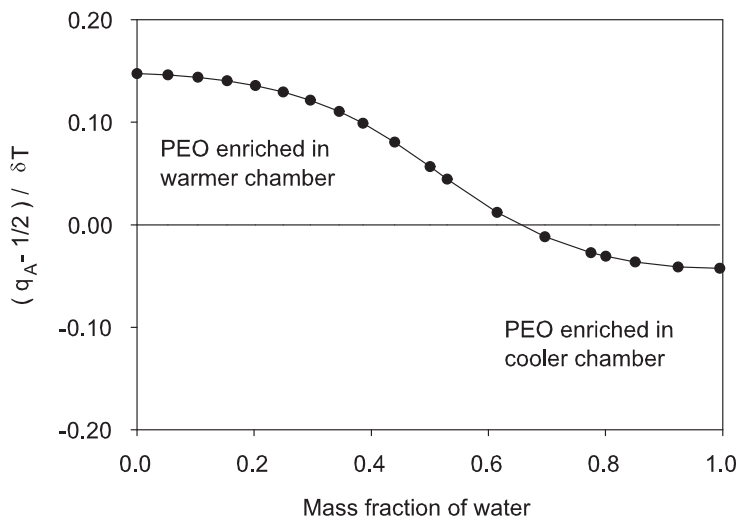

FIG. 5: Excess probability to find the PEO chain in the warmer chamber A as a function of water content of the solution. Calculations were performed for the compositions indicated by the symbols; the symbol size is larger than the uncertainty due to discrete occupation number discussed in Appendix B

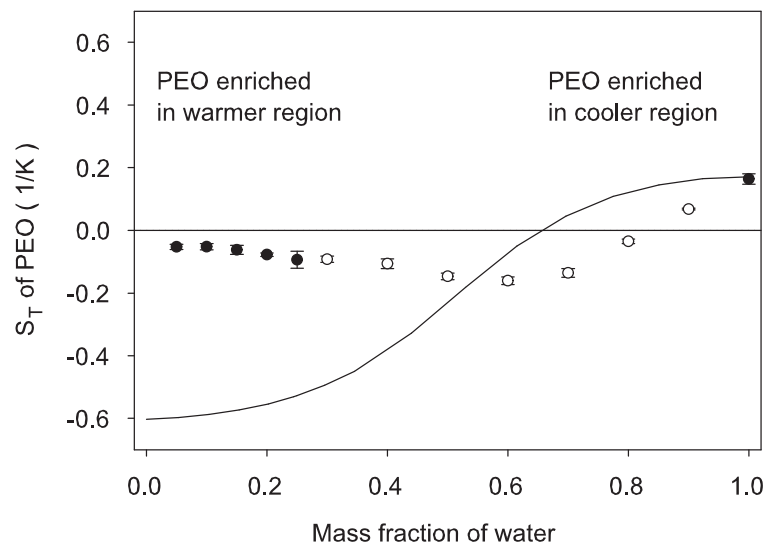

FIG. 6: Soret coefficient of PEO in mixtures of ethanol and water at room temperature and atmospheric pressure. The filled symbols represent experimental data by de Gans et al ${ }^{10}$, the open symbols represent preliminary experimental results by Kita et $a l^{32}$, the line is the result of our lattice model calculations.

for a large range of $\delta T$ values and used $\delta T=10^{-4} \mathrm{~K}$ throughout the calculations. Furthermore, since the probability to find the polymer in the warmer chamber is independent of polymer concentration, we may choose the PEO content of the solution. As discussed in Appendix [B] a low polymer concentration corresponds to a large lattice size, which reduces the uncertainty due to integer occupation numbers but increases the computation time. We found that the PEO concentration of $5 \mathrm{~g} / \mathrm{L}$ used in the experiments represents a satisfactory compromise. The results presented in Figs. [5] pertain to room temperature $(293 \mathrm{~K})$, atmospheric pressure (0.1 $\mathrm{MPa})$, and a PEO concentration of $5 \mathrm{~g} / \mathrm{L}$.

In Fig. [5 we present values for the excess probabil- 


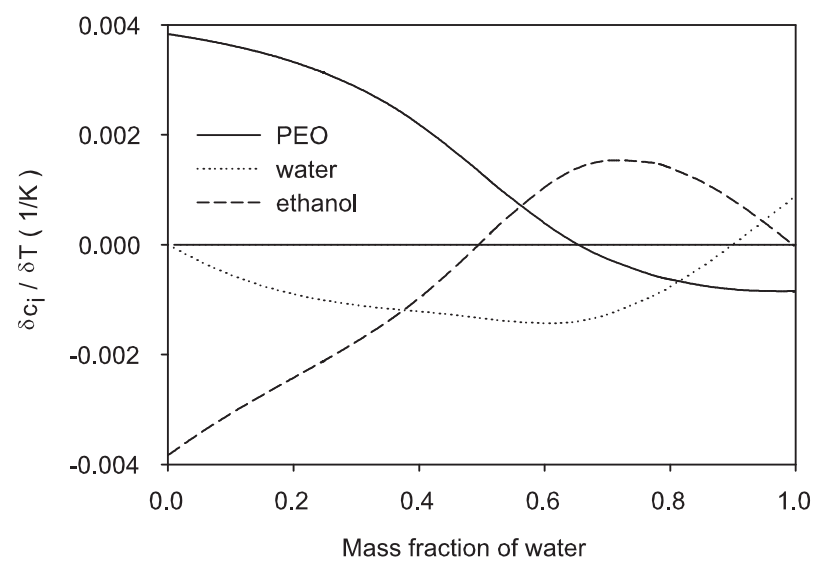

FIG. 7: Composition differences $\delta c_{i}=c_{i, \mathrm{~A}}-c_{i, \mathrm{~B}}$ between chambers $\mathrm{A}$ and $\mathrm{B}$ for the three components (PEO, ethanol, water) as a function of water content of the solution. The compositions of the chambers were calculated from Eq. 33 and divided by the temperature difference $\delta T=T_{\mathrm{A}}-T_{\mathrm{B}}=$ $10^{-4} \mathrm{~K}$.

ity $q_{\mathrm{A}}-1 / 2$ as calculated from Eq. (34). In Fig. 6] we present the corresponding values for the Soret coefficient of PEO calculated according to Eqs. (33) and (4). For comparison, we include experimental data by Wiegand and coworkers ${ }^{10.32}$. Both experiment and theory show a change in sign of the Soret coefficient as the water content of the solution is increased. For low water concentrations of the solution, the polymer is more likely to be found in the higher temperature chamber; for high water concentrations the opposite is true. Differences between theory and experiments are most pronounced at low water concentrations, where our calculations overestimate the Soret effect. This is a consequence of our choosing a mixed interaction parameter $\epsilon_{\mathrm{ps}}$ that emulates for short chains the poor solvent conditions that long PEO chains experience in ethanol.

Fig. 7 shows the composition differences $\delta c_{i}=c_{i, \mathrm{~A}}-$ $c_{i, \mathrm{~B}}$ between chambers $\mathrm{A}$ and $\mathrm{B}$ divided by the temperature difference $\delta T$ for the three components (PEO, water, ethanol) as a function of water content of the solution. For PEO in a single solvent, ethanol or water, the composition difference $\delta c_{\mathrm{p}}$ of PEO is balanced by the composition difference of the solvent. In general, the three composition differences add up to zero, $\delta c_{\mathrm{p}}+\delta c_{\mathrm{w}}+\delta c_{\mathrm{s}}=0$. The composition of the solvent is generally different in the hot and cold chamber. For a solution with mass fraction of water $c_{\mathrm{w}} \simeq 0.49$, for example, there is no difference in ethanol content of the solution of the warm and cold chamber, while water is enriched in the cold chamber. Considering the ethanol content of the solvent, $\bar{c}_{\mathrm{S}}=c_{\mathrm{s}} /\left(c_{\mathrm{S}}+c_{\mathrm{w}}\right)$, in each of the chambers, we find in our calculations that the solvent in the warm chamber is always slightly richer in ethanol than the solvent in the cold chamber. Furthermore, there is a range of water contents, approximately $10 \%$ to $35 \%$ by weight,

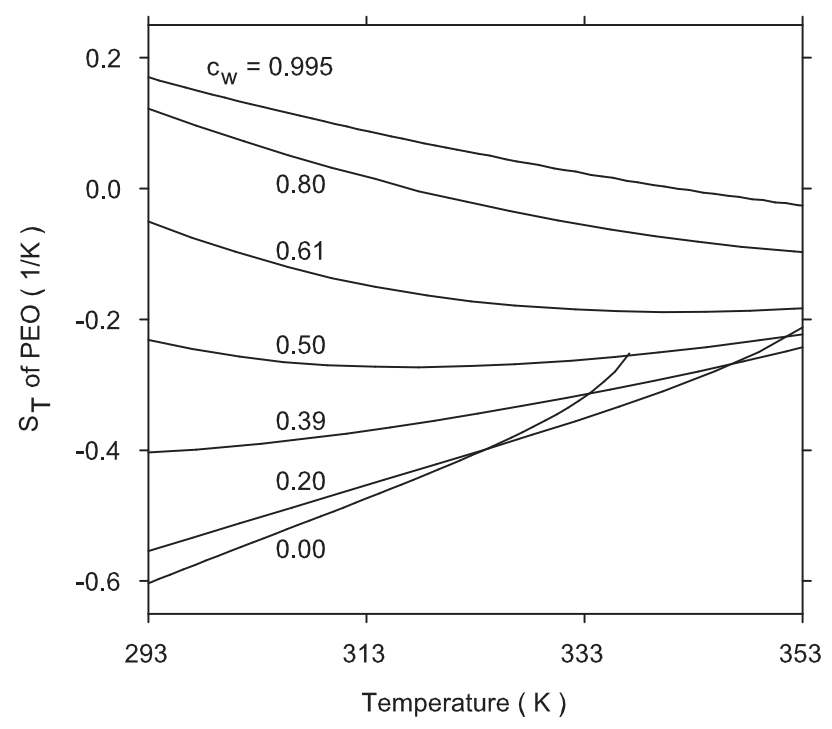

FIG. 8: Soret coefficient of PEO in mixtures of ethanol and water at atmospheric pressure as a function of temperature for several solvent compositions. The PEO concentration is $5 \mathrm{~g} / \mathrm{L}$ at $293 \mathrm{~K}$.

where the difference in solvent composition $\bar{c}_{\mathrm{s}, \mathrm{A}}-\bar{c}_{\mathrm{s}, \mathrm{B}}$ between the two chambers is approximately constant at $\left(\bar{c}_{\mathrm{s}, \mathrm{A}}-\bar{c}_{\mathrm{s}, \mathrm{B}}\right) / \delta T=2.5 \times 10^{-4} \mathrm{~K}^{-1}$.

Our results for solvent composition reflect the approximations in our calculations, in particular the random mixing approximation for solvent-solvent interactions. When we perform calculations on ethanol-water mixtures without polymer, we find a Soret coefficient for water that is positive for all compositions and decreases monotonously from $0.0069 \mathrm{~K}^{-1}$ for ethanol-rich mixtures to $0.0054 \mathrm{~K}^{-1}$ for water rich mixtures. Experimental data 14.15 .33 , on the other hand, show the Soret coefficient of water to change sign with increasing water content from around $0.006 \mathrm{~K}^{-1}$ at $50 \mathrm{wt} . \%$ to $-0.008 \mathrm{~K}^{-1}$ at 95 wt. $\%$

In Fig. 8 we present results for the temperature dependence of the Soret coefficient of PEO for several solvent compositions. For high water concentration, the Soret coefficient is positive at room temperature, decreases with increasing temperature and changes sign at a temperature that depends on the water content of the solution. For ethanol-rich solutions, the Soret coefficient is negative at room temperature and increases with increasing temperature. At the highest ethanol concentrations, an upturn of the $S_{\mathrm{T}}$ versus temperature curves is visible. This signals the onset of evaporation, which occurs at increasingly higher temperatures for solutions with larger water content. A comparison of Figs. [6 and Fig. 8 with the chain-dimension graphs Fig. [ (a) and (b) shows a correlation between solvent quality and thermodiffusion. In general, as the solvent quality increases, indicated by an increase in chain dimensions, the Soret coefficient becomes more positive. 


\section{DISCUSSION}

In this work, we have presented a two-chamber latticemodel approach to determine Soret coefficients of polymer solutions. A dilute polymer solution is represented by a simple cubic lattice occupied by a single polymer chain, solvent particles and, in the case of a compressible solvent, voids. Exact enumeration results for an isolated chain allow us to construct partition functions for the polymer-solvent system without invoking a random mixing approximation for contacts with polymer sites. Interactions between solvent particles, on the other hand, are evaluated in a random-mixing approximation. Enumeration results are also used to calculate the radius of gyration of the chain in solution, which allows us to monitor the solvent quality of the solution. In order to investigate thermodiffusion, we assume that the lattice is divided into two non-interacting sublattices of equal size that are maintained at slighty different temperatures. For a given occupation of the sublattices, the partition function of the combined system is a product of the canonical partition functions of the sublattices. We consider all possible distributions of polymer chain and solvent particles among the sublattices. The sum of states of the system is calculated by adding up the total partition functions for all distributions, while average quantities are calculated by performing the appropriate weighted sums. The Soret coefficient of the polymer is determined from the difference between the average polymer concentration in the warm and cold chambers. As in the earlier work on heat of transport (cf. Denbigh ${ }^{23}$ ) kinetic energy contributions are neglected in our calculations. However, we do not approximate the heat of transport by a difference in potential energy. Instead, the probability to find the polymer in the warmer of the two chambers can be related to a difference in average internal energy that reflects both enthalpic and entropic contributions.

We considered first a lattice model for a dilute solution of a polymer in an incompressible, single-component solvent. In this case, the canonical partition function of a short chain $\left(N_{\mathrm{c}}=17\right)$ in solution and the sum of states for the two-chamber system are calculated exactly. We derived a simple relationship between the probability to find the polymer in the warmer chamber and the internalenergy difference $U_{\text {nop }}-U_{\text {pol }}$, where $U_{\text {nop }}$ represents the internal energy of a solvent filled lattice and $U_{\text {pol }}$ is the internal energy of a lattice containing both solvent and polymer. The resulting expression for the Soret coefficient shows how our approach relates to earlier work using the heat of transport concept. It also illustrates that it is important to go beyond a random mixing approximation when thermal diffusion in polymer solutions is discussed. In agreement with experimental results for dilute solutions, ${ }^{28}$ the calculated Soret coefficients are independent of concentration. Unfortunately, the short chains considered in this work do not allow us to investigate the experimentally observed ${ }^{28}$ scaling behavior of $S_{\mathrm{T}}$ with molecular mass. We are currently working on a simula- tion approach that allows us to treat longer chains ${ }^{34}$ and will investigate the scaling laws in the near future. Our results presented in Fig. 3] show that both positive and negative Soret coefficients are realized in the incompressible case and that the sign of $S_{\mathrm{T}}$ need not be the same for all temperatures. A comparison with Fig. 11 reveals a correlation between Soret coefficients and chain dimensions for two of the three cases presented; the probability to find the polymer in the cooler chamber increases as the solvent quality increases. This trend appears to be typical and, as discussed below, may provide an explanation why negative Soret coefficients for polymer solutions are so rarely observed.

In order to describe PEO chains in a mixed solvent of ethanol and water, we extended the simple lattice model by introducing two types of solvent particles and adding voids to account for compressibility. Hydrogen bonding between PEO and water molecules is modeled through an orientational degree of freedom of the water particles. One of the six faces of an elementary cube representing a water particle has strongly attractive interactions with PEO sites, while the PEO interactions of the remaining five faces are the same as those between ethanol and PEO. The lattice model for dilute solutions of PEO in ethanol/water mixtures has eleven system-dependent parameters that are determined from static properties as described in Appendix $\mathrm{A}$ and summarized in Table 1 With these parameters, the model reproduces some of the important thermodynamic properties of the system. In particular, for PEO in mixed solvents, the solvent quality as monitored by the radius of gyration increases as the water content of the solution increases ${ }^{10.11}$. Similarly, increasing the temperature increases the solvent quality for mixtures with low water content. For mixtures with high water content, on the other hand, increasing the temperature reduces the solvent quality in agreement with observations on PEO in water (cf. Ref. 30).

Our two-chamber approach allows us to calculate Soret coefficients of PEO for given temperature, pressure and composition of the solvent. As expected for dilute solutions,$\stackrel{28}{r}$ the results are independent of the polymer concentration. In qualitative agreement with experimental data of De Gans et al. ${ }^{10.11}$ and Kita et al. ${ }^{32}$, the calculated Soret coefficients are negative for solutions with low water content and positive for solutions with high water content. While we do not expect quantitative agreement between our simple lattice model calculations and experimental data, we would like to discuss some future work that may help us gain a better understanding of thermodiffusion in this system. The evaluation of Soret coefficients with the aid of Eq. (4) is valid only when pressure differences between the chambers can be neglected. We observe very small differences between the pressures in the two chambers and do not expect them to contribute significantly, but we are investigating means to correct for this effect. Interactions between different polymer chains are not considered in our calculations, which apply to dilute solutions. However, the polymer solutions 
used in the experiments $\frac{10,11,32}{1}$ were probably in the semidilute regime. In typical semi-dilute solutions, the magnitude of the Soret coefficient decreases with increasing concentration ${ }^{28}$ It will be interesting to investigate the effect of chain-chain interactions on negative thermodiffusion coefficients. Significant thermal diffusion of the solvent particles is an issue that complicates the comparison between theory and experiment for intermediate water concentrations ${ }^{32}$ Eq. (7) for the Soret effect in ternary mixtures suggests that several diffusion coefficients need to be considered carefully for an experimental determination of the Soret coefficient in this regime. Hydrogen bonding between solvent molecules may also affect the thermal diffusion of the polymer. Our current calculations account for specific interactions only between PEO and water molecules. We will extend our model to account for hydrogen bonds between solvent molecules and investigate how it affects the Soret coefficient of the polymer.

Finally, we have also investigated the temperature dependence of the Soret coefficient of PEO and found that a change in temperature may induce a change in sign of $S_{\mathrm{T}}$. A comparison of the results for the radius of gyration of the chain and the values of the Soret coefficients reveals a close relationship between the solvent quality and the partitioning of the polymer between the chambers. In general, the Soret coefficient of the polymer becomes more positive as the solvent quality increases. A typical experiment on polymers in good solvents is thus expected to yield positive Soret coefficients. We expect negative Soret coefficients to be observed for polymers that would be insoluble were it not for specific interactions between solvent molecules and sites on the polymer. It appears that both polymer systems for which negative Soret coefficients have been observed, the solutions of PEO in a mixed ethanol/water solvent ${ }^{10,11,32}$ and the solution of poly(vinyl alcohol) in water, 9.35 belong to this category.

\section{Acknowledgments}

We would like to thank S. Wiegand and M. P. Taylor for many helpful discussions and S. Wiegand and her coworkers for making experimental data available to us prior to publication. Financial support through the National Science Foundation (NSF DMR-0103704), the Research Corporation (CC5228), and the Petroleum Research Fund (PRF \#364559 GB7) is gratefully acknowledged.

\section{APPENDIX A: DETERMINATION OF SYSTEM-DEPENDENT PARAMETERS FOR PEO IN ETHANOL WATER MIXTURES}

Our lattice model for PEO in ethanol/water mixtures has eleven system-dependent parameters. Seven of these parameters describe the thermodynamics of the pure components while the remaining four are needed for the description of the mixtures.

For each of the pure components (ethanol, water, PEO) we estimate interaction energies $\epsilon_{i i}$ and the volume $v_{0}$ per lattice site from a comparison of tabulated values ${ }^{36.37}$ for the density and the thermal expansion coefficient at standard temperature and pressure with values calculated from lattice-fluid equations of state ${ }^{31.38 .39}$, which treat nearest neighbor contacts in random mixing approximation. For water and ethanol we employ a simple lattice fluid model, the Sanchez and Lacombe equation-of-state applied to monomers, 31.38

$$
P=\frac{1}{2 v_{0}} z \epsilon \phi^{2}-\frac{R T}{v_{0}} \ln (1-\phi),
$$

where $R$ is the ideal gas constant and $v_{0}$ is the volume per mole of lattice sites. $\phi$ is the filling fraction of the lattice and related in this work to the mass density $\rho$ by

$$
\phi=\frac{s v_{0} \rho}{M},
$$

where $M$ is the molecular mass of the component and $s$ is a scale factor that is discussed below. Since the tabulated values for the properties of $\mathrm{PEO}^{37}$ correspond to large molecular mass, we use an equation of state by Costas and Sanctuary ${ }^{39}$ in the limit of infinite chain length

$$
\begin{aligned}
P= & \frac{1}{2 v_{0}} z \epsilon\left(\frac{(z-2) \phi}{z-2 \phi}\right)^{2}-\frac{R T}{v_{0}} \ln (1-\phi) \\
& +\frac{z R T}{2 v_{0}} \ln \left(1-\frac{2}{z} \phi\right)
\end{aligned}
$$

Comparing calculated and tabulated values of thermodynamic properties for each substance individually, we arrive at three different values for the volume $v_{0}$. For a description on a common lattice, we chose a value for $v_{0}$ and scale factors $s_{i}$ that allow us to reproduce the tabulated densities at standard conditions and obtain reasonable estimates for the thermal expansion coefficients. The resulting values are presented in Table — The scale factor for ethanol is chosen as $s_{\mathrm{s}}=1$ and implies that each ethanol molecule occupies one lattice site. The scale factors for water and PEO are smaller than unity. For water, there are $1 / s_{\mathrm{w}} \approx 3$ molecules associated with each site. For PEO, our short chains $\left(N_{\mathrm{c}}=17\right)$ correspond to physical chains with $N_{\mathrm{c}} / s_{\mathrm{p}} \approx 27$ repeat units. Since each repeat unit has a molecular mass of $M_{\text {mono }}=44.1 \mathrm{~g} / \mathrm{mol}$, the molecular mass of our chains is $M_{\mathrm{p}}=N_{\mathrm{c}} M_{\mathrm{mono}} / s_{\mathrm{p}} \approx 1187 \mathrm{~g} / \mathrm{mol}$.

The only additional parameters required for the description of mixtures of the three substances are the mixed interaction energies. Since we are using a very simple model to describe the solvent, we employ Berthelot's geometric mean combining rule $\epsilon_{\mathrm{ws}}=-\sqrt{\epsilon_{\mathrm{ww}} \epsilon_{\mathrm{ss}}}$ for interactions between ethanol and water. This approximation leads to deviations between calculated and tabulated $^{36}$ values for the density of less than $2 \%$ for all compositions at a temperature of $293 \mathrm{~K}$ and a pressure 
TABLE I: System-dependent parameters for the $\mathrm{PEO} /$ ethanol/water system

\begin{tabular}{l|l|l}
\hline \hline \multicolumn{1}{l|}{ lattice site volume } & $v_{0}=5.255 \times 10^{-5} \mathrm{~m}^{3} / \mathrm{mol}$ & \\
ethanol & $\epsilon_{i j}$ in $\mathrm{J} / \mathrm{mol}$ & scale factors \\
water & $\epsilon_{\mathrm{ss}}=-2306$ & $s_{\mathrm{s}}=1$ \\
$\mathrm{PEO}$ & $\epsilon_{\mathrm{ww}}=-3306$ & $s_{\mathrm{w}}=0.3362$ \\
& $\epsilon_{\mathrm{pp}}=-1153$ & $s_{\mathrm{p}}=0.6318$ \\
ethanol/water & mixed interactions & \\
$\mathrm{PEO} /$ water & $\epsilon_{\mathrm{ws}}=-\sqrt{\epsilon_{\mathrm{ww}} \epsilon_{\mathrm{ss}}}$ & \\
& $\epsilon_{\mathrm{pw}, \mathrm{n}}=2660$ & \\
PEO/ethanol & $\epsilon_{\mathrm{pw}, \mathrm{s}}=-8020$ & \\
\hline \hline
\end{tabular}

TABLE II: Comparison of tabulated ${ }^{36.37}$ and calculated values for the density $\rho$ and thermal expansion coefficient $\alpha$ at a temperature of $293 \mathrm{~K}$ and a pressure of $0.1 \mathrm{MPa}$

\begin{tabular}{l|l|l|l|l}
\hline \hline & \multicolumn{2}{|c|}{$\rho\left(\mathrm{kg} / \mathrm{m}^{3}\right)$} & \multicolumn{2}{c}{$\alpha\left(\mathrm{K}^{-1}\right)$} \\
& tabulated & calculated & tabulated & calculated \\
ethanol & 789 & 788.8 & $1.43 \times 10^{-3}$ & $1.79 \times 10^{-3}$ \\
water & 998.2 & 998.5 & $2.10 \times 10^{-4}$ & $3.24 \times 10^{-4}$ \\
PEO & 1125 & 1125.0 & $7.2 \times 10^{-4}$ & $3.83 \times 10^{-4}$ \\
\hline \hline
\end{tabular}

of $0.1 \mathrm{MPa}$. A better description of ethanol and water can be achieved when specific interactions between the molecules are included. We are currently working on such an extension of our model.

With $\epsilon_{\mathrm{ss}}$ and $\epsilon_{\mathrm{pp}}$ fixed, the mixed interaction energy $\epsilon_{\mathrm{ps}}$ determines the solubility of the polymer in ethanol. The miscibility of polymers is strongly dependent on chain length. Unfortunately, solubility data for the short chains of our calculations $\left(M_{\mathrm{p}} \approx 1187 \mathrm{~g} / \mathrm{mol}\right)$ are not available. The long PEO chains $(M \approx 236,000 \mathrm{~g} / \mathrm{mol})$ employed in the experiments ${ }^{10}$ are insoluble in ethanol at room temperature and become soluble only for temperatures above $323 \mathrm{~K}$. We obtained an estimate for $\epsilon_{\mathrm{ps}}$ for long PEO chains with the aid of the Born-Green-Yvon lattice model of Lipson and coworkers ${ }^{40,41,42}$. Employing the pure component parameters determined above, we adjusted $\epsilon_{\mathrm{ps}}$ until the calculated phase transition temperature was near $323 \mathrm{~K}$. The resulting value is $\epsilon_{\mathrm{ps}}=-1141 \mathrm{~J} / \mathrm{mol}$. This value makes ethanol an excellent solvent for the short chains, in agreement with experimental observations, but, unfortunately, it does not allow us to use our short-chain calculation to explore the effect of changing solvent quality with temperature and solvent composition. In order to find a more suitable interaction parameter, we use the radius of gyration squared, $R_{\mathrm{g}}^{2}$, as an indicator for solvent quality. We estimate a value of $\epsilon_{\mathrm{ps}}$ from the condition that the calculated value of $R_{\mathrm{g}}^{2}$ at a temperature of $323 \mathrm{~K}$ is equal to $R_{\mathrm{g}}^{2}\left(\theta^{*}\right)=4.13$, the chain dimensions of an isolated chain at the $\theta$ temperature of the infinite chain. This value for $\epsilon_{\mathrm{ps}}$ is included in Table and used in our calculations. Fig. 4 (b) includes a graph of calculated chain dimensions as a function of temperature for PEO in ethanol. A comparison with Fig. 1] shows that the chains are not collapsed at room temperature, indicating that the short chains are soluble in ethanol. However, as in the case of the long chains, solvent quality improves with increasing temperature over a large temperature range.

The interactions between $\mathrm{PEO}$ and water are described by two interaction parameters. Following Jeppesen and Kremer $^{29}$ the non-specific interactions $\epsilon_{\mathrm{pw} ; \mathrm{n}}$ are assumed to correspond to bad-solvent conditions and are approximated here by the value for the ethanol-PEO interactions. The specific (hydrogen-bonding) interactions $\epsilon_{\mathrm{pw} ; \mathrm{s}}$ are strongly attractive. As the remaining free parameter, they determine the solubility of PEO in mixtures of ethanol and water. Experiments suggest ${ }^{10}$ that the transition between poor and good solvent conditions occurs for mass fractions of water between $5 \%$ and $10 \%$. We estimate a value for $\epsilon_{\mathrm{pw} ; \mathrm{s}}$ from the condition that the calculated value of $R_{\mathrm{g}}^{2}$ in a mixture with $c_{\mathrm{w}}=0.1$ is equal to $R_{\mathrm{g}}^{2}\left(\theta^{*}\right)=4.13$.

\section{APPENDIX B: DETERMINATION OF LATTICE SIZE AND OCCUPATION NUMBERS}

In order to calculate properties of PEO solutions at given temperature, pressure, and composition, the corresponding lattice size $N$ and occupation numbers $N_{i}$, $i \in\{p, s, w\}$ have to be determined. In the results presented here, we start at room temperature, $T=293 \mathrm{~K}$, and atmospheric pressure, $P \approx 0.1 \mathrm{MPa}$, and specify the PEO concentration as mass per unit volume. This sets the lattice size, since the lattice contains a single polymer chain occupying $N_{\mathrm{c}}=17$ lattice sites

$$
N=\frac{M_{\mathrm{p}}}{v_{0}} / \rho_{\mathrm{p}},
$$

where $M_{\mathrm{p}}=N_{\mathrm{c}} M_{\text {mono }} / s_{\mathrm{p}} \approx 1187 \mathrm{~g} / \mathrm{mol}$ is the molecular mass of the chains and $\rho_{\mathrm{p}}$ is the PEO concentration in $\mathrm{g} / \mathrm{L}$. If two solvents are present, we specify the occupation number for one them, say water $\left(N_{\mathrm{w}}\right)$. The remaining $N-N_{\mathrm{c}}-N_{\mathrm{w}}$ lattice sites are then divided between ethanol $\left(N_{\mathrm{S}}\right)$ and voids $\left(N_{\mathrm{v}}\right)$ so that the pressure calculated according to Eq. (26) is approximately equal to atmospheric pressure. Since the lattice occupation numbers are integers, the targeted pressure value cannot be reached exactly. We use an iterative algorithm that identifies occupation numbers $N_{\mathrm{s}}$ and $N_{\mathrm{v}}$ corresponding to the closest calculated pressure above the target pressure. The uncertainty introduced into our results by the integer nature of the occupation numbers decreases as the size of the lattice increases. Since the number of lattice sites is set by the PEO concentration, and since our results for the Soret coefficient of $\mathrm{PEO}$ are independent of its concentration, large lattices can be used to reduce the uncertainty in the calculated values. Unfortunately, the computation time grows rapidly with increasing lattice size, so that a compromise has to be found. In this 
work, we use $\rho_{\mathrm{p}}=5 \mathrm{~g} / \mathrm{L}$ at $T=293 \mathrm{~K}$ and target pressure $P=0.1 \mathrm{MPa}$. This corresponds to a lattice size of $N=4516$ sites and yields calculated pressure values from $0.13 \mathrm{MPa}$ for ethanol rich mixtures to $0.4 \mathrm{MPa}$ for water rich mixtures. In order to perform calculations at elevated temperature or pressure, we retain the occupation numbers for the filled sites, $N_{\mathrm{c}}, N_{\mathrm{w}}$, and $N_{\mathrm{s}}$, but change the total number of lattice sites by adding or removing voids to reach the desired conditions.

The concentration of each of the components in the system is related to the lattice occupation through

$$
\rho_{i}=\frac{m_{i}}{N v_{0}}, \quad \sum_{i} \rho_{i}=\rho, \quad i \in\{s, w, p\}
$$

where

$$
m_{\mathrm{s}}=\frac{N_{\mathrm{s}} M_{\mathrm{s}}}{s_{\mathrm{s}}}, m_{\mathrm{w}}=\frac{N_{\mathrm{w}} M_{\mathrm{w}}}{s_{\mathrm{w}}}, m_{\mathrm{p}}=\frac{N_{\mathrm{c}} M_{\mathrm{mono}}}{s_{\mathrm{p}}},
$$

and $M_{\mathrm{s}}=46.07 \mathrm{~g} / \mathrm{mol}$ and $M_{\mathrm{w}}=18.0 \mathrm{~g} / \mathrm{mol}$ are the molecular masses of ethanol and water, respectively. The mass fractions $c_{i}$ of the components are given by

$$
c_{i}=\rho_{i} / \rho \text {. }
$$

* Electronic address: jutta@physics.uakron.edu

1 S. R. de Groot and P. Mazur, Non-equilibrium thermodynamics (Dover, New York, 1984).

2 R. Haase, Thermodynamics of irreversible processes (Addison-Wesley, Reading, MA, 1969).

${ }^{3}$ H. J. V. Tyrrell, Diffusion and Heat Flow in Liquids (Butterworths, London, 1961).

4 J. E. Powers, in New Chemical Engineering Separation Techniques, edited by H. M. Schoen (Interscience, New York, 1962), chap. 1, pp. 1-98.

5 W. Köhler and S. Wiegand, eds., Thermal Nonequilibrium Phenomena in Fluid Mixtures (Springer, Berlin, 2002).

${ }^{6}$ C. A. Rue and M. E. Schimpf, Anal. Chem. 66, 4054 (1994).

7 W. Köhler and R. Schäfer, Adv. Polym. Sci. 151, 1 (2000).

8 P. M. Shiundu and J. C. Giddings, J. Chromatogr. A 715, 117 (1995).

9 M. Giglio and A. Vendramini, Phys. Rev. Lett. 38, 26 (1977).

10 B.-J. de Gans, R. Kita, B. Müller, and S. Wiegand, J. Chem. Phys., in press.

11 B.-J. de Gans, S. Wiegand, and J. Luettmer-Strathmann, submitted to Phys. Rev. Lett.

12 P. F. van Velden, H. P. G. van der Voort, and C. J. Gorter, Physica 12, 151 (1946).

13 I. Prigogine, L. de Brouckère, and R. Amand, Physica 16, 851 (1950).

14 P. Kolodner, H. Williams, and C. Moe, J. Chem. Phys. 88, 6512 (1988).

15 K. J. Zhang, M. E. Briggs, R. W. Gammon, and J. V. Sengers, J. Chem. Phys. 104, 6881 (1996).

16 J. P. Larre, J. K. Platten, and G. Chavepeyer, Int. J. Heat Mass Transfer 40, 545 (1997).

17 S. Chapman and T. G. Cowling, The mathematical theory of non-uniform gases (Cambridge University, Cambridge, UK, 1970), 3rd ed.

18 B. Hafskjold, in Thermal nonequilibrium phenomena in fluid mixtures, edited by W. Köhler and S. Wiegand (Springer, Berlin, 2002), pp. 3-23.

19 K. Wirtz, Die Naturwissenschaften 27, 369 (1939).

${ }^{20}$ K. Wirtz, Physikalische Zeitschrift 44, 221 (1943).

${ }^{21}$ K. Wirtz and J. W. Hiby, Physikalische Zeitschrift 44, 369
(1943)

22 I. Prigogine, L. de Brouckère, and R. Amand, Physica 16, 577 (1950).

${ }^{23}$ K. G. Denbigh, Trans. Faraday Soc. 48, 1 (1952).

24 A. M. Nemirovsky, K. F. Freed, T. Ishinabe, and J. F. Douglas, Physics Letters A 162, 469 (1992).

25 A. M. Nemirovsky, K. F. Freed, T. Ishinabe, and J. F. Douglas, J. Stat. Phys. 67, 1083 (1992).

26 M. Doi, Introduction to Polymer Physics (Clarendon, Oxford, 1996).

27 H. Meirovitch and H. A. Lim, J. Chem. Phys. 92, 5144 (1990).

28 J. Rauch and W. Köhler, Phys. Rev. Lett. 88, 185901 (2002).

29 C. Jeppesen and K. Kremer, Europhys. Lett. 34, 563 (1996).

30 E. E. Dormidontova, Macromolecules 35, 987 (2002).

31 S. M. Lambert, Y. Song, and J. M. Prausnitz, in Equations of state for fluids and fluid mixtures, edited by J. V. Sengers, R. F. Kayser, C. J. Peters, and H. J. White Jr. (Elsevier, Amsterdam, 2000), chap. 14, pp. 523-588.

32 R. Kita and S. Wiegand, to be published.

33 J. F. Dutrieux, J. K. Platten, C. G., and M. M. Bou-Ali, J. Phys. Chem. B 106, 6104 (2002).

34 J. Luettmer-Strathmann and M. Boiwka, in preparation.

35 D. Eagland and N. J. Crowther, Faraday Symp. Chem. Soc. 17, 141 (1982).

36 R. C. West, ed., Handbook of Chemistry and Physics (Chemical Rubber, Boca Raton, FL, 1978), 59th ed.

37 D. W. van Krevelen, Properties of Polymers (Elsevier, Amsterdam, 1972).

38 I. C. Sanchez and R. H. Lacombe, J. Phys. Chem. 80, 2352 (1976).

39 M. Costas and B. C. Sanctuary, J. Phys. Chem. 85, 3153 (1981).

40 J. E. G. Lipson and P. K. Brazhnik, J. Chem. Phys. 98, 8178 (1993).

41 J. Luettmer-Strathmann and J. E. G. Lipson, Fluid Phase Equilib. 150-151, 649 (1998).

42 J. Luettmer-Strathmann, J. A. Schoenhard, and J. E. G. Lipson, Macromolecules 31, 9231 (1998). 